\title{
ENHANCING A LAPAROSCOPY TRAINING SYSTEM WITH AUGMENTED REALITY VISUALIZATION
}

\author{
Hao Jiang \\ Siqing Xu \\ Andrei State \\ Fan Feng \\ Henry Fuchs \\ Department of Computer Science \\ University of North Carolina \\ Chapel Hill, NC, USA \\ Minsik Hong \\ Jerzy Rozenblit \\ Dept. of Electrical and Computer Engineering \\ University of Arizona \\ Tucson, AZ, USA \\ mshong@email.arizona.edu,jr@ece.arizona.edu \\ \{haojiang,xsq4525,andrei,fan8,fuchs\}@ cs.unc.edu
}

\begin{abstract}
We report work in progress towards a system for laparoscopy training that is enhanced with an augmented reality display. The system can be used to perform peg-transfer training tasks using a Microsoft Hololens AR device, with full 6 degrees of freedom (DoF) shared presence with the pegboard workspace. This mode is in contrast to the conventional visualization, whose views are solely through a 2D or stereo laparoscope. In order to achieve this enhanced visualization, the system extracts the pose of the triangular prism being manipulated, and of the laparoscopic instruments with which the user manipulates the prism. These two objects are added to a CAD model of a (fixed) pegboard, and all the objects are visualized within the AR display. In the near future we expect to conduct user studies measuring accuracy and time-to-completion of this peg-transfer task using these new enhancements and compare it to conventional visualization techniques.
\end{abstract}

Keywords: laparoscopic, augmented reality, visualization

\section{INTRODUCTION}

Laparoscopic surgery is a widely used and effective minimally invasive surgery procedure. Since laparoscopic surgery minimizes incisions, compared to conventional open surgery, it reduces pain and injury to the patient, and reduces recovery time. However, laparoscopic surgery is difficult to perform since there is constrained space inside the patient's body, which severely limits both the views of the surgical site and manipulations therein. Thus, extensive training is required.

Many laparoscopy training systems have been developed, from simple "box" trainers to trainers that integrate sophisticated computer simulations and haptic feedback (Schijven and Jakimowicz 2003, Botden and Jakimowicz 2009).

We use a laparoscopic trainer system similar to CAST system (Rozenblit et al. 2014). This system provides both visual and haptic feedback. Given a realistic training environment (e.g., 3D printed organ models), a shortest and collision-free path is generated for instrument navigation. While a trainee performs a task, a performance evaluator collects movement data and quantifies competency objectively. Also, visual and force 
guidance are provided in order to teach proper maneuvering of surgical instruments based on previously generated recommended trajectories.

We report our initial results of enhancing this trainer with a head-mounted AR display, with which the surgeon can visualize the laparoscopic scene and instruments from arbitrary viewpoints while naturally moving around the (internal) surgical scene. We expect that this capability, combined with the haptics feedback guidance, will provide more natural visualization, comprehension, and thus yield improved task performance.

To enable the free-viewpoint visualization, the internal 3D surgical scene must be extracted, modeled and dynamically recreated. The resulting $3 \mathrm{D}$ visualization can then be observed on a variety of display types, among them conventional fixed display, head-mounted virtual reality, or head-mounted augmented reality. We chose head-mounted AR (in contrast to VR) because it allows users to observe their hands, the equipment, and the rest of the operating environment, including the patient and other medical personnel. Figure 1 gives an overview of our system in which a user is performing the peg-transfer task and visualizing the scene in a head-mounted AR display.

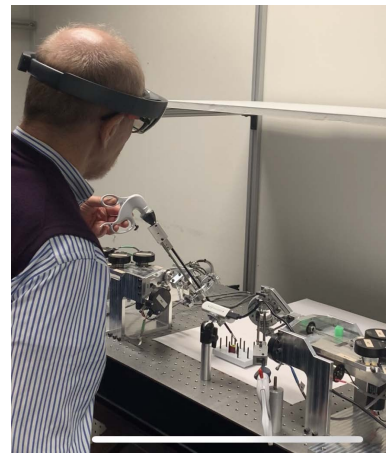

(a)

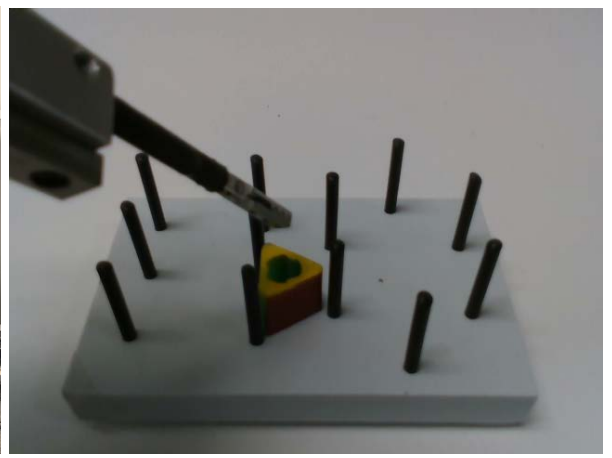

(b)

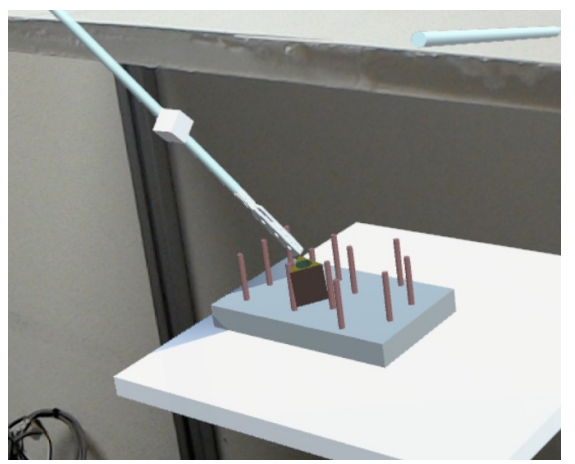

(c)

Figure 1: (a) A user is grabbing the triangular prism in our training system. (b) Laparoscopic camera view. (c) Augmented reality view (from free viewpoint) in Microsoft HoloLens.

The rest of the paper is organized as follows: Section 2 introduces related work in visualization and reconstruction in laparoscopic surgery. Section 3 describes our system. Section 4 shows numerical results of the tracking algorithm and some initial and intuitive results from our visualization system. Section 5 concludes our work and describes possible improvement.

\section{RELATED WORK}

There is a class of immersive virtual reality based laparoscopy training systems which are similar to our system (Mohammadi et al. 2010, Huber et al. 2018). The purpose of these systems is to provide surgeons with novel training content which is analogous to real laparoscopic surgery. Surgeons can perform an operation and see the operating room scene as if they were in a real laparoscopic surgery. However, in such systems surgeons still visualize the inner body using a conventional 2D laparoscopic display, while our system allows surgeons to visualize the instrument and key components involved in the surgery in a 3D AR display. In some other systems (Botden et al. 2008, Lahanas et al. 2015), AR simulators were used to present rich surgical training scenes, or to provide virtual training setups using a $2 \mathrm{D}$ monitor. 


\subsection{Visualization in Laparoscopic Surgery}

In traditional laparoscopic surgery and laparoscopy training systems, surgeons visualize the scene and the instruments through a single 2D color camera inserted into the body of patient. However, since the viewing position and direction of the camera is very constrained, it is not convenient for surgeons to perform certain operations. Researchers have been working to improve 3D visualization in the past decades. Feuerstein et al. developed a system allowing visualization of the internal scene from multiple or moving perspectives (Feuerstein et al. 2008). Fuchs et al. proposed to visualize laparoscopic surgery in a see-through AR display (Fuchs et al. 1998). Such 3D visualization has been demonstrated to be effective and can reduce operation time in both laparoscopic surgery and training systems (Bhayani and Andriole 2005, Korndorffer Jr et al. 2005, Tanagho et al. 2012, Cologne et al. 2015, Velayutham et al. 2016). However, in most such 3D visualization systems, the surgeon is still limited to visualizing the scene from the camera's viewpoint(s). Our system frees the viewing position of the surgeon from the laparoscope camera, which makes the procedure more natural and more akin to open surgery.

\subsection{Reconstruction in Laparoscopic Surgery}

Reconstruction in laparoscopic surgery is critical for augmented visualization since surgeons need to make decisions and perform procedures based on reconstruction results. However, high quality reconstruction in laparoscopic surgery is challenging since the scene contains small and shiny objects, large deformations and wet, textureless surfaces. Besides, in such a small working volume, it is difficult to deploy advanced sensors or engineer illumination conditions.

In the past decades, work described in several papers addressed these problems. One class of methods use structured light to project known patterns onto the scene and reconstruct the scene from the deformation of the pattern (Fuchs et al. 1998, Ackerman et al. 2002, Clancy et al. 2015, Lin et al. 2015). Another class of methods use time-of-flight sensors to measure light travel duration between camera and scene, then calculate the distance (Tatar et al. 2004, Penne et al. 2009), thus constructing a depth map. More recent work performs stereo and fusion from a pair of cameras in order to obtain a per-pixel depth map (Lo et al. 2008, Mountney and Yang 2010, Röhl et al. 2011, Collins and Bartoli 2012, Edgcumbe et al. 2013). These methods could be applied as we apply our system to real laparoscopic surgery scenarios.

Currently our system works in a constrained training environment with simple and known objects. As a consequence, we apply pose estimation algorithms to objects with known shape. Pose estimation is a class of algorithms which predict 6D object pose from RGB or RGBD input. There are several related recent publications in the computer vision community. PoseNet (Kendall et al. 2015) uses convolutional structure to predict the pose of camera. PoseCNN (Xiang et al. 2017) jointly performs object detection, segmentation and pose estimation. Instead of directly predicting 6D pose, (Tekin et al. 2017) predict the projection of keypoints. From the 2D projection of the keypoints and corresponding 3D keypoints they obtain the pose by solving the PnP (Perspective-n-Point) problem.

\section{METHODS}

In this section, we describe our laparoscopy training system components. As already mentioned in the Introduction, we start with a system similar to CAST system (Rozenblit et al. 2014) and add 3D AR visualization to it. For our first user experiences, we are developing the capability to support a classic peg-transfer task (Way et al. 1995, Peng et al. 2017), in which the user transfers a single donut-like triangular prism made of rubber from one peg to another, using either one or two laparoscopic grasping instruments. For this task, the simple 3D "surgical" scene consists of a) a pegboard, b) a triangular prism, c) two laparoscopic 
grasping instruments, and d) a "laparoscope" camera. These physical components are shown in Figure 2. Not shown is the Microsoft HoloLens, the AR system worn by the user.

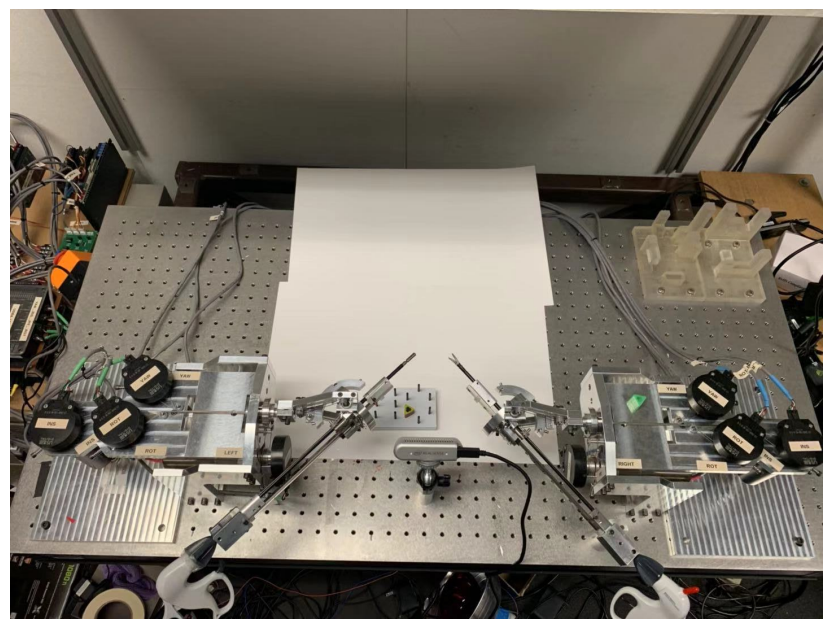

Figure 2: Our experimental system.

The system diagram is shown in Figure 3. During system operation, the user views a real-time rendering of the objects in the surgical site: the pegboard, the triangular prism, and the two manipulators. The 3D CAD models of each of these have been manually constructed and pre-installed within the rendering module in the HoloLens. During system operation, only the positions and orientations of the triangular prism and of the two manipulators need to be updated. The pose (position and orientation) of the triangular prism is computed from the camera's image; the poses of the manipulators are obtained from the laparoscopic fixture's optical encoders. (The pegboard is attached to the base of the fixture and thus cannot move.) The poses of the triangular prism and manipulators are sent from the PCs to the HoloLens via UDP over WiFi. These parts are explained in the following subsections: triangular prism reconstruction (Section 3.1), instrument tracking (Section 3.2), and AR visualization (Section 3.3).

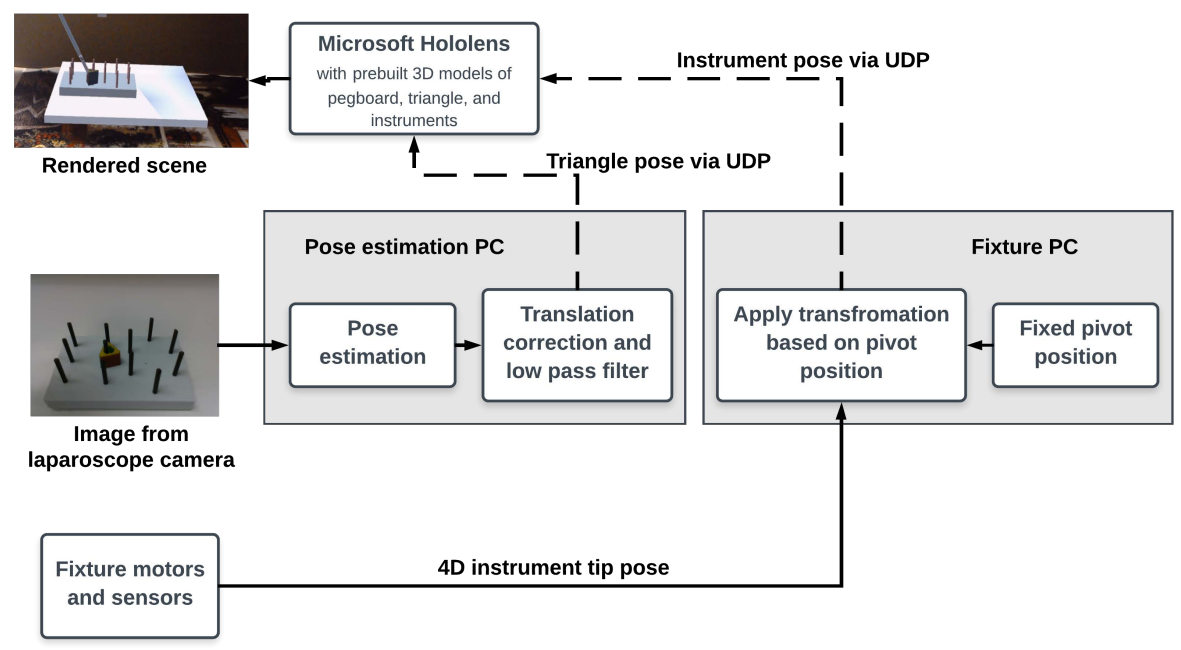

Figure 3: System diagram. 


\subsection{Triangular Prism Reconstruction}

Unlike methods which output depth map using stereo or deep learning (Mahjourian et al. 2018), we extract the pose of the triangular prism (a 6D pose vector) from a single RGB image captured by the "laparoscope" camera. After determining the pose of the triangular prism, the virtual model of the triangular prism is rendered by the HoloLens. We train a deep learning model which predicts the pose of the triangular prism given an RGB image, at 10 frames per second.

\subsubsection{Dataset}

There are several public datasets for object pose estimation (Feuerstein et al. 2008, Brachmann et al. 2014) but none of them contains objects similar to the triangular prism used here. As a result, we built our own dataset. Since labelling the ground truth pose of photographed triangular prisms is time-consuming, we generated a synthetic dataset via graphics rendering and directly applied the training results to the real data (Tobin et al. 2017).

Our dataset uses a manually created triangular prism model which has the same shape and color as the real one, for which the pose must be estimated (See Figure 4). We generate random poses for the triangular prism model and generate virtual images that simulate the camera, with the same extrinsic and intrinsic parameters as our real camera. Then the real background captured by camera is added to each rendered image to form a training input image. The pose and segmentation map of the triangular prism are recorded as ground truth data. We also provide some meta data, such as camera parameters and object bounding box. In our dataset, occlusion and lighting effects are not (yet) taken into account, but we still get reasonably accurate results as we will show later. We generated 4800 samples for training and 200 samples for validating.

\subsubsection{Network}

We adopted the network from the PoseCNN work (Xiang et al. 2017) which was tested on a large and comprehensive dataset known as the YCB video dataset (Xiang et al. 2017). PoseCNN is a deep learning architecture which takes monocular RGB image as input and jointly outputs object bounding box, segmentation map and 6D pose. PoseCNN uses VGG16 (Simonyan and Zisserman 2014) to extract the feature of the image; feature extraction is followed by three branches. The segmentation branch predicts a segmentation map which segments the triangular prism against the background. Each pixel in segmentation map indicates the category that pixel belongs to ( 1 for triangular prism and 0 for background). The translation branch predicts a vertex map where each pixel is a vector of the normalized distances from current pixel to the center of the triangular prism in 3 dimensions. PoseCNN then use Hough voting to extract bounding box and translation vector of the triangular prism. Finally in the rotation branch, the feature map with in the bounding box is cropped, pooled and forwarded into 3 fully connected layers (FCLs) to get the quaternion which represents rotation of the triangular prism.

The loss function contains 4 terms. Similar to the original PoseCNN paper, a cross entropy loss is applied to the segmentation map and a smooth 11 loss is applied to the vertex map. However, we change the loss of pose to 12 loss of the quaternion since we don't have symmetry objects in our experiment and the 12 loss is a stronger supervision compared to the average points distance in the original PoseCNN paper. A regularization term is added to reduce overfitting. 


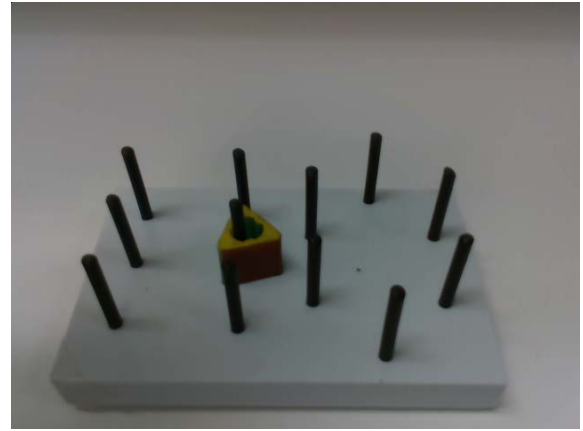

(a)

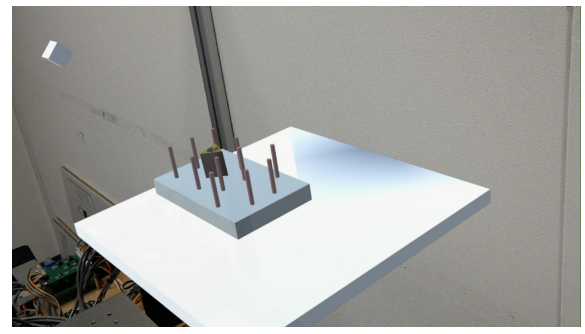

(c)

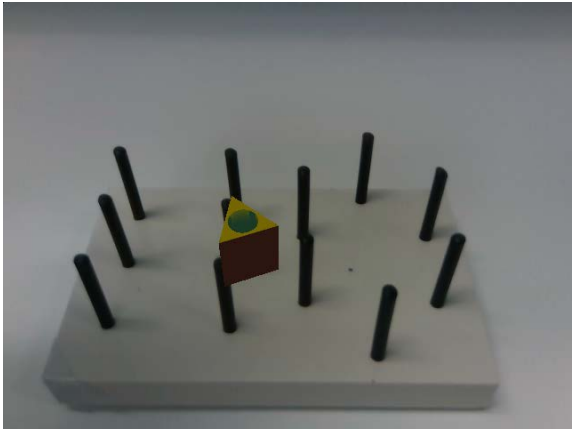

(b)

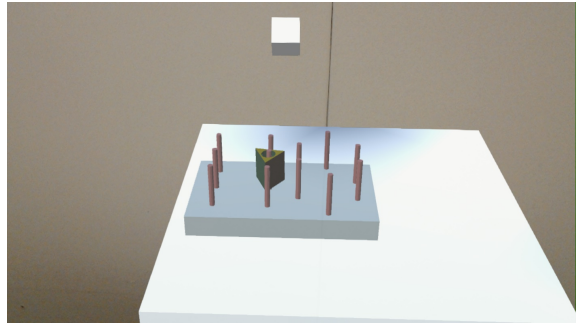

(d)

Figure 4: Real and reconstructed scenes. (a) Image from camera. (b) Synthetic reconstructed triangular prism repositioned over camera's image (without occlusion). (c, d) Two views of the AR scene displayed to HoloLens user, user having moved the entire synthetic scene, on a synthetic white board, to a comfortable height just below eye level.

\subsubsection{Post Processing}

Laparoscopy training requires very accurate and robust reconstruction of the triangular prism. However, as a statistical method, the error in pose estimation cannot be well controlled, therefore it does not satisfy this requirement. In order to improve the performance, we added some post processing steps to refine the pose estimate.

First, we observe that compared with rotation error, translation error is more critical in our training system. A few millimeters translation error will cause the 3D model of triangular prism to indicate incorrectly that the user has made a wrong decision. To alleviate this problem, we correct the translation as follows: we extract the segmentation map of triangular prism and calculate a translation offset vector that maximizes the overlap between the projection of the 3D model of the triangular prism and its segmentation map.

In addition, we observe random jitter errors of the triangular prism in a video sequence, caused by random rotation and translation errors. We added a low pass filter to quaternion and translation vector to smooth the pose results.

\subsection{Instrument Tracking}

Our trainer has two mechanical fixtures which are designed for installation of various real surgical instruments (e.g., needle drivers or graspers). Each fixture has a gimbal to allow four-degree-of-freedom movement (i.e., yaw, pitch, roll, and insertion) by mimicking a trocar's functionality. Also, the fixture has three motors to provide force feedback for yaw, pitch, and insertion motions, and four optical encoders to track 
the instrument tip. We use a forward kinematics model and an optimization algorithm similar to a previously described one (Hong et al. 2017) for the instrument tip position and orientation estimation. The 3D position for yaw, pitch and insertion movements and the roll can be represented as a $4 \mathrm{D}$ vector and are computed in real time.

\subsection{AR Visualization}

We created the 3D model of the key components including pegboard, triangular prism, instruments, and camera in Unity and put them up in the Microsoft HoloLens. The visualization application receives the poses of triangular prism and instruments and renders them. For the triangular prism, the $6 \mathrm{D}$ pose is converted to a transformation matrix and applied to the 3D model. The instrument pose is represented as a rotation around a fixed pivot point and insertion length. The system receives the tip points of the instruments and predefined rotation pivot points. Then rotation is computed from the direction between pivot point and tip point and insertion is computed from the distance between pivot point and tip point.

In the application, the user can move the entire scene to any location and view it from any direction. Recall that in a real training environment, the actual surgical site is covered up and thus only the synthesized views in the HoloLens are visible. Initially, we thought that users would prefer to see the synthesized scene at the location of the real surgical site, to give an illusion similar to Superman's X-ray vision. However, users seem to find it tiring to look down on the surgical site while standing up and manipulating the laparoscopic instruments, and often seem to prefer viewing the reconstructed scene at a more comfortable height close to eye level.

\section{RESULTS}

\subsection{Triangular prism Tracking}

We use the implementation in (Xiang et al. 2017) for training and testing. We trained the network for 160,000 iterations with a batch size of 2 and SGD optimizer with momentum 0.9 and initial learning rate 0.001 . We use the average magnitude of relative rotation(RR) and relative translation(RT) between predicted pose and ground truth pose as evaluation metrics.

We tested our tracking algorithm on both synthetic dataset and real dataset. For synthetic dataset, we randomly generated 200 poses and rendered the virtual triangular prism in these poses. For the real dataset, we manually labelled the pose of a sequence of 194 images. The results are shown in Table 1 . We observed that the translation errors distributed mostly perpendicular to the image plane so that the translation error along the image plane is very small (see 4 th column in Table 1). This observation motivates us to use multi-camera setting in the future.

Table 1: Results of triangular prism tracking.

\begin{tabular}{|c|c|c|c|}
\hline & average RR $($ degree$)$ & average RT $(\mathrm{cm})$ & average RT along image plane $(\mathrm{cm})$ \\
\hline synthetic data & 8.64 & 0.260 & 0.19 \\
\hline real data & 9.62 & 0.421 & 0.15 \\
\hline
\end{tabular}




\subsection{AR Visualization}

We have integrated the triangular prism pose estimation and instrument tracking into the AR visualization system. The pose estimation, with input from the color camera in an Intel RealSense D435, is running under the Ubuntu 16.04 operating system and uses a GEFORCE GTX $1080 \mathrm{Ti}$ GPU. The pose estimation time is around $0.07 \mathrm{~s}$ per frame. The instrument tracking is running on the CPU.

We show some visualization results in Figure 5 and Figure 6, where a user is transferring the triangular prism from one peg to another. We observed that the virtual instrument and triangular prism appear in roughly the same spatial relationship as their real counterparts. However, there remain some problems, such as the virtual triangular prism occasionally intersecting with the virtual pegboard, which may confuse the user.

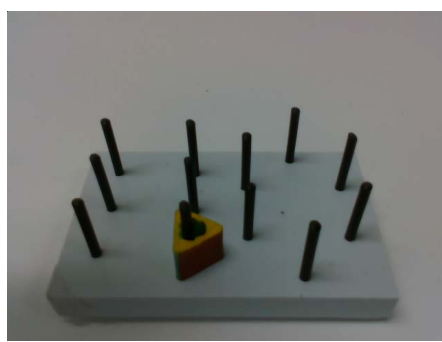

(a)

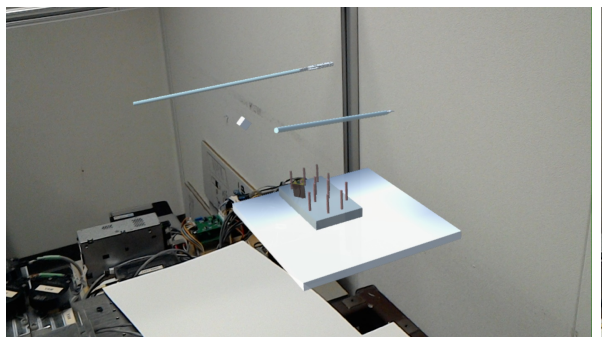

(c)

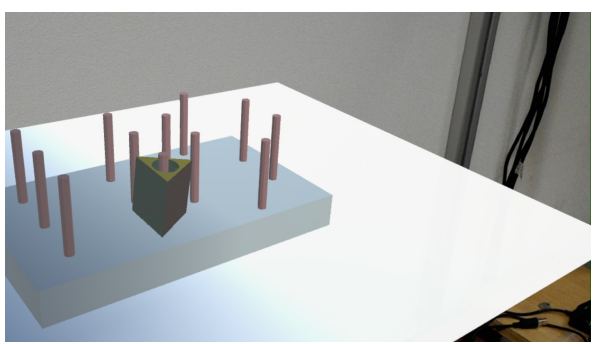

(b)

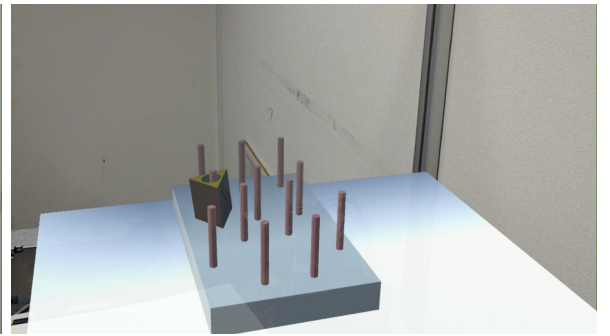

(d)

Figure 5: Reconstruction results for the triangular prism in different views during the peg-transfer task. (a) Source image from laparoscope camera. (b, c, d) Reconstructed scene in HoloLens, shown from different viewing positions.

We are currently attempting the peg-transfer task in laparoscopy training in which a user transfers the triangular prism from one peg to another while looking at the virtual scene in the HoloLens rather than at the real scene. (Recall that the real scene is blocked.) We observed that while the visualization appears to be a pleasant experience for users, the slight errors of a few $\mathrm{mm}$ between manipulator tip and triangular prism position hinder users so much that precise manipulations such as grasping the edge of the triangular prism are rarely successful. We hope to reduce the tracking error, so that these kinds of manipulations will become easy. We also plan to conduct a user study to learn whether our AR visualization can improve laparoscopy training task performance.

\section{CONCLUSION AND FUTURE WORK}

In this paper we have shown initial results of adding AR visualization to a haptic-enhanced laparoscopy training system. We use a pose estimation algorithm to reconstruct a triangular prism manipulated by an opto-mechanically tracked laparoscopic grasper. We render the scene in a Microsoft HoloLens. We have 


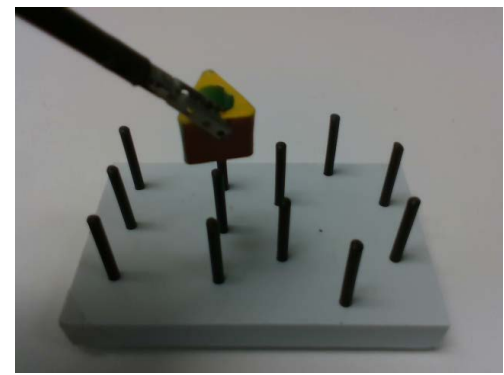

(a)

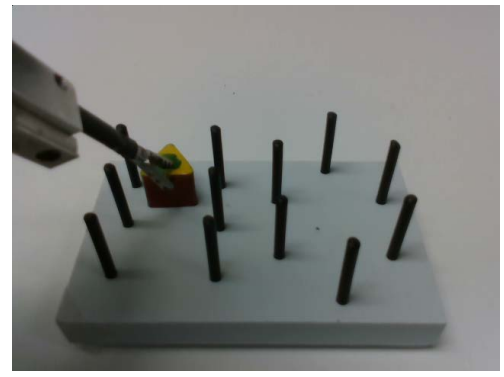

(c)

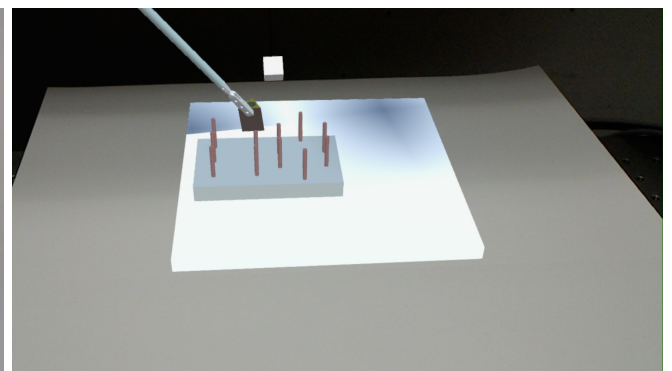

(b)

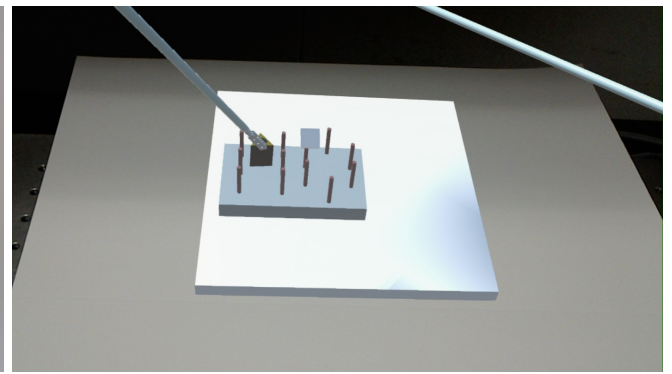

(d)

Figure 6: Reconstruction result in peg-transfer task. Left column shows source images from the laparoscope camera used for triangulr prism pose estimation. Right column shows scene displayed in the Microsoft HoloLens.

started to perform peg-transfer experiments. We show the result of successfully reconstructing triangular prism and instruments, demonstrating correct spatial relationships between instruments, triangular prism and pegboard.

In addition to improving calibration and tracking accuracy, we plan to integrate the AR visualization with haptic feedback, in order to present the preferred manipulation paths indicated by an expert teacher. We also plan to conduct user studies to determine whether the combination of AR visualization and haptic feedback influences learning effectiveness, and compare it with traditional video displays.

\section{REFERENCES}

Ackerman, J. D., K. Keller, and H. Fuchs. 2002. "Surface reconstruction of abdominal organs using laparoscopic structured light for augmented reality". In Three-Dimensional Image Capture and Applications $V$, Volume 4661, pp. 39-47. International Society for Optics and Photonics.

Bhayani, S. B., and G. L. Andriole. 2005. "Three-dimensional (3D) vision: does it improve laparoscopic skills? An assessment of a 3D head-mounted visualization system”. Reviews in urology vol. 7 (4), pp. 211.

Botden, S. M., S. N. Buzink, M. P. Schijven, and J. J. Jakimowicz. 2008. "ProMIS augmented reality training of laparoscopic procedures face validity". Simulation in healthcare vol. 3 (2), pp. 97-102.

Botden, S. M., and J. J. Jakimowicz. 2009. "What is going on in augmented reality simulation in laparoscopic surgery?”. Surgical endoscopy vol. 23 (8), pp. 1693. 
Brachmann, E., A. Krull, F. Michel, S. Gumhold, J. Shotton, and C. Rother. 2014. "Learning 6d object pose estimation using 3d object coordinates". In European conference on computer vision, pp. 536-551. Springer.

Clancy, N. T., J. Lin, S. Arya, G. B. Hanna, and D. S. Elson. 2015. "Dual multispectral and 3D structured light laparoscope". In Multimodal Biomedical Imaging X, Volume 9316, pp. 93160C. International Society for Optics and Photonics.

Collins, T., and A. Bartoli. 2012. "3d reconstruction in laparoscopy with close-range photometric stereo". In International Conference on Medical Image Computing and Computer-Assisted Intervention, pp. 634-642. Springer.

Cologne, K. G., J. Zehetner, L. Liwanag, C. Cash, A. J. Senagore, and J. C. Lipham. 2015. "Threedimensional laparoscopy: does improved visualization decrease the learning curve among trainees in advanced procedures?". Surgical laparoscopy, endoscopy \& percutaneous techniques vol. 25 (4), pp. 321-323.

Edgcumbe, P., C. Nguan, and R. Rohling. 2013. "Calibration and stereo tracking of a laparoscopic ultrasound transducer for augmented reality in surgery". In Augmented Reality Environments for Medical Imaging and Computer-Assisted Interventions, pp. 258-267. Springer.

Feuerstein, M., T. Mussack, S. M. Heining, and N. Navab. 2008. "Intraoperative laparoscope augmentation for port placement and resection planning in minimally invasive liver resection". IEEE Transactions on Medical Imaging vol. 27 (3), pp. 355-369.

Fuchs, H., M. A. Livingston, R. Raskar, D. Colucci, K. Keller, A. State, J. R. Crawford, P. Rademacher, S. H. Drake, and A. A. Meyer. 1998. "Augmented reality visualization for laparoscopic surgery". In International Conference on Medical Image Computing and Computer-Assisted Intervention, pp. 934943. Springer.

Hong, M., K.-S. Peng, V. Lundine, and J. W. Rozenblit. 2017. "Laparoscopic instrument tip position estimation for visual and haptic guidance in the computer assisted surgical trainer". In Systems, Man, and Cybernetics (SMC), 2017 IEEE International Conference on, pp. 3083-3088. IEEE.

Huber, T., T. Wunderling, M. Paschold, H. Lang, W. Kneist, and C. Hansen. 2018. "Highly immersive virtual reality laparoscopy simulation: development and future aspects". International journal of computer assisted radiology and surgery vol. 13 (2), pp. 281-290.

Kendall, A., M. Grimes, and R. Cipolla. 2015. "Posenet: A convolutional network for real-time 6-dof camera relocalization". In Proceedings of the IEEE international conference on computer vision, pp. 29382946.

Korndorffer Jr, J. R., J. B. Dunne, R. Sierra, D. Stefanidis, C. L. Touchard, and D. J. Scott. 2005. "Simulator training for laparoscopic suturing using performance goals translates to the operating room". Journal of the American College of Surgeons vol. 201 (1), pp. 23-29.

Lahanas, V., C. Loukas, N. Smailis, and E. Georgiou. 2015. "A novel augmented reality simulator for skills assessment in minimal invasive surgery”. Surgical endoscopy vol. 29 (8), pp. 2224-2234.

Lin, J., N. T. Clancy, and D. S. Elson. 2015. "An endoscopic structured light system using multispectral detection". International journal of computer assisted radiology and surgery vol. 10 (12), pp. 19411950.

Lo, B., A. J. Chung, D. Stoyanov, G. Mylonas, and G.-Z. Yang. 2008. "Real-time intra-operative 3D tissue deformation recovery". In Biomedical Imaging: From Nano to Macro, 2008. ISBI 2008. 5th IEEE International Symposium on, pp. 1387-1390. IEEE. 
Mahjourian, R., M. Wicke, and A. Angelova. 2018. "Unsupervised Learning of Depth and Ego-Motion from Monocular Video Using 3D Geometric Constraints". In Proceedings of the IEEE Conference on Computer Vision and Pattern Recognition, pp. 5667-5675.

Mohammadi, Y., M. A. Lerner, A. S. Sethi, and C. P. Sundaram. 2010. "Comparison of laparoscopy training using the box trainer versus the virtual trainer". JSLS: Journal of the Society of Laparoendoscopic Surgeons vol. 14 (2), pp. 205.

Mountney, P., and G.-Z. Yang. 2010. "Motion compensated SLAM for image guided surgery". In International Conference on Medical Image Computing and Computer-Assisted Intervention, pp. 496-504. Springer.

Peng, K. S., M. Hong, and J. Rozenblit. 2017. "Image-based object state modeling of a transfer task in simulated surgical training". In Proceedings of the Symposium on Modeling and Simulation in Medicine, pp. 6. Society for Computer Simulation International.

Penne, J., K. Höller, M. Stürmer, T. Schrauder, A. Schneider, R. Engelbrecht, H. Feußner, B. Schmauss, and J. Hornegger. 2009. "Time-of-flight 3-D endoscopy". In International Conference on Medical Image Computing and Computer-Assisted Intervention, pp. 467-474. Springer.

Röhl, S., S. Bodenstedt, S. Suwelack, H. Kenngott, B. P. Mueller-Stich, R. Dillmann, and S. Speidel. 2011. "Real-time surface reconstruction from stereo endoscopic images for intraoperative registration". In Medical Imaging 2011: Visualization, Image-Guided Procedures, and Modeling, Volume 7964, pp. 796414. International Society for Optics and Photonics.

Rozenblit, J. W., C. Feng, M. Riojas, L. Napalkova, A. J. Hamilton, M. Hong, P. Berthet-Rayne, P. Czapiewski, G. Hwang, J. Nikodem et al. 2014. "The computer assisted surgical trainer: design, models, and implementation". In Proceedings of the 2014 Summer Simulation Multiconference, pp. 30. Society for Computer Simulation International.

Schijven, M., and J. Jakimowicz. 2003. "Virtual reality surgical laparoscopic simulators". Surgical Endoscopy And Other Interventional Techniques vol. 17 (12), pp. 1943-1950.

Simonyan, K., and A. Zisserman. 2014. "Very deep convolutional networks for large-scale image recognition". arXiv preprint arXiv:1409.1556.

Tanagho, Y. S., G. L. Andriole, A. G. Paradis, K. M. Madison, G. S. Sandhu, J. E. Varela, and B. M. Benway. 2012. "2D versus 3D visualization: impact on laparoscopic proficiency using the fundamentals of laparoscopic surgery skill set". Journal of Laparoendoscopic \& Advanced Surgical Techniques vol. 22 (9), pp. 865-870.

Tatar, F., J. Mollinger, J. Bastemeijer, and A. Bossche. 2004. "Time of flight technique used for measuring position and orientation of laparoscopic surgery tools". In Sensors, 2004. Proceedings of IEEE, pp. 1480-1483. IEEE.

Tekin, B., S. N. Sinha, and P. Fua. 2017. "Real-time seamless single shot 6D object pose prediction". arXiv preprint arXiv:1711.08848 vol. 2.

Tobin, J., R. Fong, A. Ray, J. Schneider, W. Zaremba, and P. Abbeel. 2017. "Domain randomization for transferring deep neural networks from simulation to the real world". In Intelligent Robots and Systems (IROS), 2017 IEEE/RSJ International Conference on, pp. 23-30. IEEE.

Velayutham, V., D. Fuks, T. Nomi, Y. Kawaguchi, and B. Gayet. 2016. "3D visualization reduces operating time when compared to high-definition 2D in laparoscopic liver resection: a case-matched study". Surgical endoscopy vol. 30 (1), pp. 147-153.

Way, L., S. Bhoyrul, and T. Mori. 1995. Fundamentals of laparoscopic surgery. Churchill Livingstone.

Xiang, Y., T. Schmidt, V. Narayanan, and D. Fox. 2017. "Posecnn: A convolutional neural network for 6d object pose estimation in cluttered scenes". arXiv preprint arXiv:1711.00199. 


\section{AUTHOR BIOGRAPHIES}

HAO JIANG is a PhD student in Computer Science Department at University of North Carolina at Chapel Hill. His research interest lies in 3D computer vision, graphics and related AR/VR applications. He received his B.E. in Electronic Information Engineering at University of Science and Technology of China. His email address is haojiang@cs.unc.edu.

SIQING XU is a senior undergraduate student at University of North Carolina at Chapel Hill. His majors are Computer Science and Mathematics. He has been an undergraduate research assistant working at Computer Science Department of University of North Carolina at Chapel Hill for two and a half years. His research interest are 3D reconstruction and Augmented Reality. His email address is xsq4525@cs.unc.edu.

MINSIK HONG is a Ph.D. candidate at the University of Arizona. He received a Master of Science degree in Electrical and Computer Engineering from POSTECH, Republic of Korea. His research interests are robotics, control system, fuzzy theory, and modeling and simulation for medical devices. His email address is mshong@email.arizona.edu.

ANDREI STATE is a Senior Research Scientist in the UNC Department of Computer Science and cofounder of InnerOptic Technology Inc., which creates medical 3D guidance technology for minimally invasive interventions. His research interests include virtual/augmented reality and display technology. He received a Dipl.-Ing. aer. degree from the University of Stuttgart (Germany) and an MS in computer science from UNC. His email address is andrei@cs.unc.edu.

FAN FENG is an undergraduate research assistant in the department of computer science at University of North Carolina at Chapel Hill. Her research interests include modeling and simulation in virtual/augmented reality. She is currently pursuing a BS degree in computer science from UNC. Her email address is fan8@live.unc.edu.

JERZY W. ROZENBLIT is University Distinguished Professor, Raymond J. Oglethorpe Endowed Chair in the Electrical and Computer Engineering (ECE) Department, with a joint appointment as Professor of Surgery in the College of Medicine at the University of Arizona. During his tenure at the University of Arizona, he established the Model-Based Design Laboratory with major projects in design and analysis of complex, computer-based systems, hardware/software codesign, and simulation modeling. He presently serves as Director of the Life-Critical Computing Systems Initiative, a research enterprise intended to improve the reliability and safety of technology in healthcare and life-critical applications. His email address is jr@ece.arizona.edu.

HENRY FUCHS is the Federico Gil Distinguished Professor of Computer Science and Adjunct Professor of Biomedical Engineering at UNC Chapel Hill. He has been active in computer graphics since the 1970s, with rendering algorithms (BSP Trees), hardware (Pixel-Planes), Office of the Future, virtual and augmented reality, tele-immersion systems and medical applications. His email address is fuchs@cs.unc.edu. 